\title{
Geometric Permutations Induced by Line Transversals through a Fixed Point*
}

\author{
Boris Aronov ${ }^{1}$ and Shakhar Smorodinsky ${ }^{2}$ \\ ${ }^{1}$ Computer and Information Science Department, Polytechnic University, \\ Brooklyn, NY 11201-3840, USA \\ aronov@poly.edu \\ ${ }^{2}$ Institute for Theoretical Computer Science, ETH Zurich, \\ IFW, CH-8092, Zurich, Switzerland \\ sshakhar@inf.ethz.ch
}

\begin{abstract}
A line transversal of a family $S$ of $n$ pairwise disjoint convex objects is a straight line meeting all members of $S$. A geometric permutation of $S$ is the pair of orders in which members of $S$ are met by a line transversal, one order being the reverse of the other.

In this note we consider a long-standing open problem in transversal theory, namely, that of determining the largest number of geometric permutations that a family of $n$ pairwise disjoint convex objects in $\mathbb{R}^{d}$ can admit. We settle a restricted variant of this problem. Specifically, we show that the maximum number of those geometric permutations to a family of $n>2$ pairwise-disjoint convex objects that are induced by lines passing through any fixed point is between $K(n-1, d-1)$ and $K(n, d-1)$, where $K(n, d)=\sum_{i=0}^{d}\left(\begin{array}{c}n-1 \\ i\end{array}\right)=\Theta\left(n^{d}\right)$ is the number of pairs of antipodal cells in a simple arrangement of $n$ great $(d-1)$-spheres in a $d$-sphere. By a similar argument, we show that the maximum number of connected components of the space of all line transversals through a fixed point to a family of $n>2$ possibly intersecting convex objects is $K(n, d-1)$.

Finally, we refute a conjecture of Sharir and Smorodinsky on the number of neighbor pairs in geometric permutations and offer an alternative conjecture which may be a first step towards solving the aforementioned general problem of bounding the number of geometric permutations.
\end{abstract}

\footnotetext{
* Work on this paper has been partially supported by a grant from the U.S.-Israeli Binational Science Foundation. The first author's work on this paper has also been supported by NSF Grant CCR-9972568 and NSF ITR Grant CCR-0081964. Part of the work was performed while he was visiting the Mathematical Sciences Research Institute, Berkeley, California. Part of the work on this paper was performed while the second author was a Ph.D student at Tel-Aviv University, under the supervision of Micha Sharir, and later a postdoctoral fellow at the Mathematical Sciences Research Institute, Berkeley, California.
} 


\section{Introduction}

In this paper we refer to a closed convex set as an object. Let $S$ be a family of objects in $\mathbb{R}^{d}$. A line $\ell$ is said to be a transversal for $S$ if it intersects every member of $S$. If $S$ is finite and consists of pairwise-disjoint objects, then a line transversal for $S$ induces two linear orderings on $S$ - the two orders in which the members of $S$ are met by $\ell$, corresponding to the two orientations of $\ell$. (We normally do not distinguish between the two permutations corresponding to opposite orientations of a line transversal.) Katchalski et al. [4] were the first to study such orderings and called them geometric permutations.

Let $g(S)$ denote the number of geometric permutations of a family $S$ of pairwisedisjoint objects and let $g_{d}(n):=\max g(S)$, with the maximum taken over all such families $S$ of size $n$ in $\mathbb{R}^{d}$. The following asymptotic bounds on $g_{d}(n)$ are known: $g_{2}(n)$ $=2 n-2$ [3], and in higher dimensions $g_{d}(n)$ is $\Omega\left(n^{d-1}\right)$ [5] and $O\left(n^{2 d-2}\right)$ [11]. Hence, for $d \geq 3$ there remains a wide gap between the known upper and lower bounds. Better bounds are known for some restricted families of objects (see, e.g., [1], [6], [7], [10], [14], and [15]). In particular, $g_{d}(n)=\Theta\left(n^{d-1}\right)$ for families of balls. Recent surveys of results on transversals and geometric permutations can be found in [9] and [12].

We start with some notation. Let $\mathbb{S}^{d}$ be the unit $d$-sphere centered at the origin $O$ in $\mathbb{R}^{d+1}$. We identify directed lines through the origin in $\mathbb{R}^{d}$ with points on $\mathbb{S}^{d-1}$ and undirected lines with pairs of antipodal points there. Let $K(n, d)$ be the maximum number of pairs of antipodal $d$-cells in an arrangement of $n$ great $(d-1)$-spheres in $\mathbb{S}^{d}$; it is known that $K(n, d)=\sum_{i=0}^{d}\left(\begin{array}{c}n-1 \\ i\end{array}\right)$ (see, e.g., [2] and [13]). The maximum is achieved precisely when the arrangement is simple, meaning that any set of $d$ of the great spheres have precisely two points in common.

\section{Line Transversals through the Origin}

In [10] it was shown that the lower bound of $\Omega\left(n^{d-1}\right)$ on $g_{d}(n)$ can be attained by families of balls. Furthermore, the lower bound construction described there is such that all geometric permutations are induced by lines passing through the origin. (Note that, although we are interested in transversals passing through an arbitrary fixed point, it is sufficient to consider the case where the point is the origin $O$. This is the convention we follow hereafter.) In this section we examine this restricted version of the problem. Namely, if $S$ is a family of pairwise-disjoint objects, let $\hat{g}(S)$ be the number of geometric permutations induced by line transversals of $S$ that pass through the origin. Put $\hat{g}_{d}(n):=$ $\max \hat{g}(S)$, with the maximum taken over all such families $S$ of $n$ objects in $\mathbb{R}^{d}$. Below we determine the exact expression for $\hat{g}_{d}(n)$, up to a lower-order term. We start with a simple lemma, whose proof we include for completeness.

Lemma 1. Let $r$ and $r^{\prime}$ be two non-collinear rays sharing an endpoint and meeting all members of a family $S$ of objects in $\mathbb{R}^{d}$. Then

(a) if the objects in $S$ are pairwise disjoint, $r$ and $r^{\prime}$ meet them in the same order, and

(b) any ray in the wedge between $r$ and $r^{\prime}$ also meets all members of $S$. 


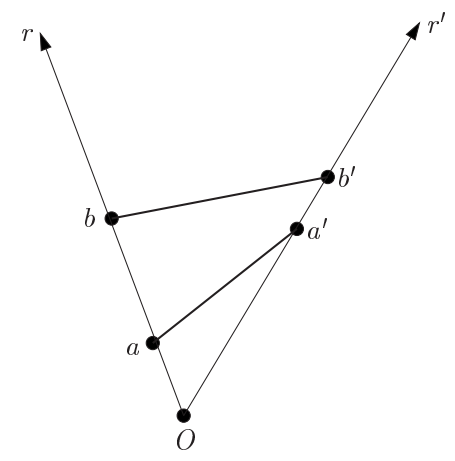

Fig. 1. The two rays $r$ and $r^{\prime}$ meet the members of $S$ in the same order; the cross section by the plane $\pi$ spanned by $r, r^{\prime}$ is shown.

Proof. (a) Consider the plane $\pi$ spanned by $r$ and $r^{\prime}$; for each object $A \in S$ pick a straight-line segment connecting some two points $a, a^{\prime}$ where $a \in A \cap r$ and $a^{\prime} \in A \cap r^{\prime}$. By convexity, we have $a a^{\prime} \subseteq A \cap \pi$. If $r$ and $r^{\prime}$ meet some two objects $A, B$ in different order then the corresponding co-planar segments $a a^{\prime} \subseteq A$ and $b b^{\prime} \subseteq B$ must cross, contradicting the assumption that $A$ and $B$ are disjoint (see Fig. 1 for an illustration).

For part (b) observe that, using the same notation, a ray in the wedge has to meet $a a^{\prime} \subseteq A$, for all $A \in S$.

Theorem 1. Consider a family $S$ of $n$ convex objects in $\mathbb{R}^{d}$.

(a) The maximum number of path-connected components of the space of lines transversal to $S$ that pass through the origin is $K(n, d-1)=\Theta\left(n^{d-1}\right)$; the objects may be unbounded or non-disjoint.

(b) If the objects are pairwise disjoint, the maximum number $\hat{g}_{d}(n)$ of geometric permutations induced by lines passing through the origin is at most $K(n, d-1)$ and at least $K(n-1, d-1)=K(n, d-1)-\Theta\left(n^{d-2}\right)$.

In both cases the lower bounds can be attained by a family of pairwise-disjoint balls; the lower bound for case (a) can be attained by a family of overlapping unit balls.

Before proceeding to the proof of the theorem, we start with an easy corollary of Lemma 1 . Any directed line transversal $\ell$ of $S$ through the origin, naturally partitions $S$ into the set $S^{-}(\ell)$ of objects lying before the origin $O$ along $\ell$ and the set $S^{+}(\ell)$ of objects met by $\ell$ after $O$. (This is not strictly a partition if some object in $S$ contains the origin -we consistently exclude it from both sets hereafter.)

Lemma 2. The set of orientations of all directed lines $\ell$ through the origin, transversal to $S$ and inducing a fixed partition $\left(S^{-}(\ell), S^{+}(\ell)\right)=\left(S^{-}, S^{+}\right)$of $S$, is a convex set in $\mathbb{S}^{d-1}$.

Proof. Let $\ell_{1}, \ell_{2}$ be two lines that induce the same partition: $\left(S^{-}\left(\ell_{1}\right), S^{+}\left(\ell_{1}\right)\right)=$ $\left(S^{-}\left(\ell_{2}\right), S^{+}\left(\ell_{2}\right)\right)$. Rotate $\ell_{1}$ into $\ell_{2}$ in the plane spanned by them through the smaller 
angle between their directions. Apply Lemma 1(b) separately to the positive rays of the two lines and the set $S^{+}\left(\ell_{1}\right)=S^{+}\left(\ell_{2}\right)$, and then to the negative rays and the set $S^{-}\left(\ell_{1}\right)=S^{-}\left(\ell_{2}\right)$, to conclude that each intermediate line $\ell$ meets all members of $S$ and induces the same partition of $S$; if an object of $S$ contains the origin, it also obviously meets $\ell$.

Proof of Theorem 1. We use a modification of the technique that was originally employed by Wenger [11] for proving the upper bound $O\left(n^{2 d-2}\right)$ on $g_{d}(n)$. We start with an upper bound for part (a). By Lemma 2, the number of connected components in question cannot exceed half the number of ordered partitions $\left(S^{-}(\ell), S^{+}(\ell)\right.$ ) of $S$ induced by some directed transversal $\ell$ of $S$. For each object $b \in S$, choose a hyperplane $h_{b}$ that separates the origin $O$ and $b$, and consider the great $(d-2)$-sphere $s_{b} \subset \mathbb{S}^{d-1}$ consisting of all orientations parallel to $h_{b}$ (namely, $s_{b}=h_{b}^{\prime} \cap \mathbb{S}^{d-1}$, where $h_{b}^{\prime}$ is $h_{b}$ translated to $O$ ). Consider the arrangement $\mathcal{A}$ of the resulting set of $n$ great spheres in $\mathbb{S}^{d-1}$. Recall that we identify directed lines through the origin with points on $\mathbb{S}^{d-1}$. Any such line corresponding to a direction in $s_{b}$ is separated from $b$ by $h_{b}$ and thus misses it. In particular, any line transversal of $S$ through the origin must lie in the complement of $\bigcup_{b \in S} s_{b}$, that is, in an open $(d-1)$-dimensional cell of the arrangement $\mathcal{A}$. Let $C$ be one such cell. We next show that if the orientations of two directed line transversals $\ell_{1}, \ell_{2}$ of $S$ belong to $C$, they induce the same $\left(S^{-}(\ell), S^{+}(\ell)\right)$ partition of $S$. This will complete the upper bound argument for part (a). Any object $b \in S$ is intersected by an oriented line $\ell$ before or after the origin (if at all), depending only on the hemisphere of $\mathbb{S}^{d-1} \backslash s_{b}$ containing the direction of $\ell$. Since $C$ is a cell of the arrangement $\mathcal{A}$ formed by the great spheres $s_{b}$, any two lines with orientation in $C$ lie in the same open hemisphere bounded by $s_{b}$, for all $b \in S$. Thus $S^{+}\left(\ell_{1}\right)=S^{+}\left(\ell_{2}\right)$ and $S^{-}\left(\ell_{1}\right)=S^{-}\left(\ell_{2}\right)$, as claimed.

Next, we argue that $\hat{g}_{d}(n) \leq K(n, d-1)$. Consider again the arrangement $\mathcal{A}$. We have seen that, for any two lines $\ell_{1}, \ell_{2}$ lying in the same cell $C$ of $\mathcal{A}, S^{+}\left(\ell_{1}\right)=S^{+}\left(\ell_{2}\right)$ and $S^{-}\left(\ell_{1}\right)=S^{-}\left(\ell_{2}\right)$. Applying Lemma $1\left(\right.$ a) to $S^{+}\left(\ell_{1}\right)$ and the positive rays of $\ell_{1}$ and $\ell_{2}$ (resp., to $S^{-}\left(\ell_{1}\right)$ and the negative rays), we conclude that the two lines meet the sets in $S^{+}\left(\ell_{1}\right)$ (resp., in $S^{-}\left(\ell_{1}\right)$ ) in the same order. Hence $\ell_{1}$ and $\ell_{2}$ induce the same geometric permutation on $S$. (Note that this argument is unaffected by the possible presence in $S$ of an object that contains the origin-we may disregard this object entirely, as it always occurs between $S^{-}(\ell)$ and $S^{+}(\ell)$ along any line transversal $\ell$.) Thus the number of geometric permutations induced by undirected lines through the origin does not exceed the maximum number of pairs of antipodal $(d-1)$-dimensional cells in the arrangement $\mathcal{A}$ of $n(d-2)$-spheres in $\mathbb{S}^{d-1}$. Therefore $\hat{g}_{d}(n) \leq K(n, d-1)$.

Finally, we describe the lower bound for both parts of the theorem. It is a variant of the construction of Smorodinsky et al. [10]. We start by constructing in $\mathbb{R}^{d}$ an arrangement of $n$ hyperplanes $\Pi=\left\{\pi_{1}, \ldots, \pi_{n}\right\}$ passing through the origin, so that the intersections of the hyperplanes with $\mathbb{S}^{d-1}$ form a simple arrangement $\mathcal{A}$ of great $(d-2)$-spheres there. Since $\mathcal{A}$ is simple and thus has $K(n, d-1)$ pairs of antipodal cells, replacing each great sphere $\sigma$ by an open $\varepsilon$-band $\sigma_{\varepsilon}$ (i.e., the locus of points on $\mathbb{S}^{d-1}$ at distance less than $\varepsilon$ from $\sigma$ ), for a sufficiently small $\varepsilon>0$, yields a collection of bands the complement of the union of which also has $K(n, d-1)$ pairs of antipodal cells. For a hyperplane $\pi$ through the origin, let $\sigma(\pi)$ denote the corresponding great sphere in $\mathbb{S}^{d-1}$ and let $\sigma_{\varepsilon}(\pi)$ denote the corresponding $\varepsilon$-band. For each hyperplane $\pi$, first place a unit ball $B_{\pi}$ 
tangent to $\pi$ at the origin and then shift it in direction orthogonal to $\pi$ and away from it just enough to guarantee that a line through the origin misses $B_{\pi}$ if and only if it makes an angle less than $\varepsilon$ with $\pi$.

The balls can be made pairwise disjoint by the method outlined in the following paragraph. The resulting collection $\left\{B_{\pi}\right\}_{\pi \in \Pi}$ demonstrates the tightness of the bound in (a). Indeed, any two lines through the origin whose orientations lie in distinct nonantipodal $(d-1)$-dimensional cells of $\mathcal{A}$ cannot induce the same partition $\left(S^{-}(\cdot), S^{+}(\cdot)\right)$, by Lemma 2 . Since every cell corresponds to some realizable ordered partition, the number of unordered realizable partitions is exactly $K(n, d-1)$.

For part (b), we repeat the above construction, with two modifications. One is that we let $O$ be one of our objects. The remaining $n-1$ are balls constructed as above, but we must make the balls pairwise disjoint. We proceed incrementally, after fixing the order of the balls arbitrarily. The first ball remains unchanged. The second is scaled with the center at the origin until it is disjoint from the first one. The third is scaled until it is disjoint from the first two. After $n-2$ re-scalings, we obtain a collection of $n-1$ pairwise-disjoint balls which are partitioned in $K(n-1, d-1)$ different ways by the origin (which is also an object in our collection) in their unordered geometric permutations induced by lines through the origin. Hence the number of such geometric permutations is at least $K(n-1, d-1)$, proving the claimed lower bound. If desired, $O$ can be replaced by a suitably chosen small ball of positive radius.

\section{Neighbors in Geometric Permutations}

Let $S$ be a family of pairwise-disjoint objects in $\mathbb{R}^{d}$. Two objects of $S$ are called (geometric-permutation) neighbors if there is a geometric permutation of $S$ in which they are consecutive [8]. Let $p(S)$ be the number of neighbor pairs in $S$ and put $p_{d}(n)=\max p(S)$, with $S$ ranging over all such families of $n$ objects in $\mathbb{R}^{d}$; trivially $p_{d}(n) \leq\left(\begin{array}{l}n \\ 2\end{array}\right)$. Sharir and Smorodinsky [8] suggested a new approach for strengthening the general upper bound on $g_{d}(n)$. Namely, they showed that the number of geometric permutations of $S$ is bounded by $K\left(p_{d}(S), d-1\right)=O\left(p_{d}(S)^{d-1}\right)$ and conjectured that $p_{d}(n)=O(n)$ in all dimensions (indeed they proved that $\left.p_{2}(n)=\Theta(n)\right)$. We disprove the conjecture in dimensions greater than two by constructing already in $\mathbb{R}^{3}$ a family of pairwise-disjoint balls with $\Omega\left(n^{2}\right)$ neighbor pairs-these pairs in fact all appear in geometric permutations realized by lines through the origin.

Theorem 2. The maximum number $p_{d}(n)$ of geometric-permutation neighbor pairs in a set of $n$ pairwise-disjoint convex objects in $\mathbb{R}^{d}$ is $\Theta(n)$ for $d=1,2$ and $\Theta\left(n^{2}\right)$ for $d>2$. The same bounds hold for families of pairwise-disjoint balls.

Proof. The statement is vacuously true in $d=1$ and has been settled by Sharir and Smorodinsky [8] for $d=2$. The upper bound of $\left(\begin{array}{l}n \\ 2\end{array}\right)$ is immediate, in all dimensions. To complete the proof it suffices to construct a lower bound in dimension three. We describe the construction of a set of pairwise-disjoint rectangles in $\mathbb{R}^{3}$ and then explain how to transform the rectangles into pairwise-disjoint balls. Our construction is related to the one used for the lower bound of Theorem 1(b) (and to the standard example of a family 


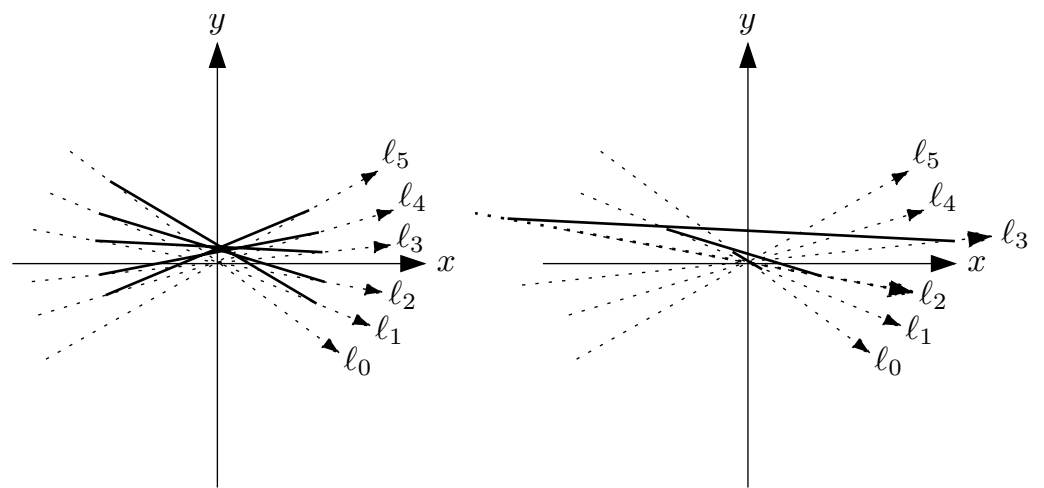

Fig. 2. A family of $k$ segments in the plane with $k-1$ geometric permutations realized by lines $\ell_{1}, \ldots, \ell_{k-1}$ through $O$. The figure on the left shows the segments unscaled, for $k=5$, while on the right the first three segments are depicted scaled to avoid intersection. The two figures are not on the same scale.

of segments in the plane with many line transversals), although we arrive at it from a different direction.

Without loss of generality, assume $n$ is even and put $n=2 k$. Consider $k+1$ lines with positive slopes strictly between minus one and one passing through the origin $O$ in the $x y$-plane. We order them by slope, $\ell_{0}, \ell_{1}, \ldots, \ell_{k}$, from smallest to largest. We treat each $\ell_{i}$ as directed from left to right; $O$ partitions it into two open rays, one negative and one positive. We construct a collection of $n$ disjoint segments $s_{1}, \ldots, s_{k}$, all meeting the positive $y$-axis; refer to Fig. 2 . The first segment $s_{1}$ connects an arbitrary point on the negative ray of $\ell_{0}$ to an arbitrary point on the positive ray of $\ell_{1}$. The second segment $s_{2}$ connects a point on the negative ray of $\ell_{1}$ to a point on the positive ray of $\ell_{2}$ which are chosen far enough from $O$ for $s_{2}$ to avoid $s_{1}$. This can be achieved by first picking points on the two rays arbitrarily, disregarding the disjointness constraint, and then scaling the resulting segment by a homothety with center $O$ and positive magnification large enough for $s_{2}$ to avoid $s_{1}$. The process is then repeated for each segment in turn, with the $i$ th segment $s_{i}$ connecting the negative ray of $\ell_{i-1}$ to the positive ray of $\ell_{i}$ while avoiding all the previously constructed segments by being "further away" from $O$.

By construction, in the range of slopes strictly between slope $\left(\ell_{0}\right)$ and slope $\left(\ell_{k}\right)$ the transversals of $\left\{s_{1}, \ldots, s_{k}\right\}$ are precisely the lines $\ell_{i}, i=1, \ldots, k-1$. (We disregard all other transversals, such as the $y$-axis.) The permutations they induce are

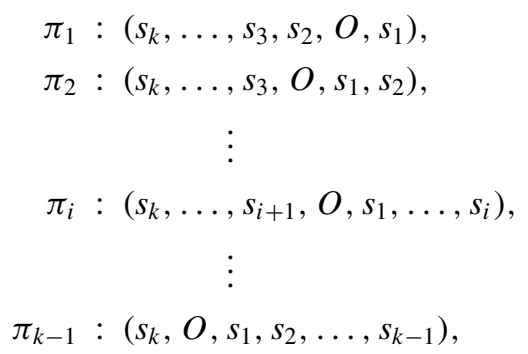


where we have indicated the positions of the origin in the permutation with an $O$ and where the (directed) permutation $\pi_{i}$ corresponds to the (directed) line $\ell_{i}$. Notice that $\pi_{i}$ ends with $s_{i}$ and has $s_{i+1}$ as the last element before the origin. This is the fact we use to construct a set with a large number of geometric-permutation neighbor pairs.

Now construct a family $R=\left\{r_{1}, \ldots, r_{k}\right\}$ of $k$ rectangles in $\mathbb{R}^{3}$, rectangle $r_{i}$ corresponding to segment $s_{i}$ and such that the following properties are satisfied:

- The orthogonal projection of $r_{i}$ to the $x y$-plane is $s_{i}$.

- Two of the edges of $r_{i}$ are parallel to the $z$-axis.

- Any line $\eta$, with $O \in \eta \subset \mathbb{R}^{3}$, that makes an angle less than $\pi / 4$ with the $x y$-plane, and such that its orthogonal projection $\eta_{x y}$ to the $x y$-plane makes an angle less than $\pi / 4$ with the $x$-axis, meets $r_{i}$.

These conditions ensure that any line $\eta$ in $\mathbb{R}^{3}$ that projects to $\eta_{x y}=\ell_{i}$ and makes an angle less than $\pi / 4$ with it, is a transversal of $R$.

We now construct a second family of rectangles, $R^{\prime}=\left\{\rho_{1}, \ldots, \rho_{k}\right\}$, as a copy of $R$ rotated by $\pi / 2$ around the $x$-axis and then scaled by a homothety with center at $O$ and a negative scaling factor of sufficiently small absolute value so as to be disjoint from $R$. The last condition can be satisfied since all rectangles of $R$ lie at a positive distance from $O$, which implies the existence of a positive-radius ball centered at $O$ and avoiding $R$. $R^{\prime}$ is scaled to be contained in this ball. The resulting $R^{\prime}$ admits the following $k-1$ geometric permutations,

$$
\begin{gathered}
\sigma_{1}:\left(\rho_{1}, O, \rho_{2}, \rho_{3}, \ldots, \rho_{k}\right), \\
\sigma_{2}:\left(\rho_{2}, \rho_{1}, O, \rho_{3}, \ldots, \rho_{k}\right), \\
\vdots \\
\sigma_{j}:\left(\rho_{j}, \ldots, \rho_{1}, O, \rho_{j+1}, \ldots, \rho_{k}\right), \\
\vdots \\
\sigma_{k-1}:\left(\rho_{k-1}, \ldots, \rho_{2}, \rho_{1}, O, \rho_{k}\right),
\end{gathered}
$$

induced by respective lines $\lambda_{j}, j=1, \ldots, k-1$, lying in the $x z$-plane. Now consider the collection $\left\{\mu_{i j}\right\}$ of $(k-1)^{2}$ lines, where $\mu_{i j}, i, j=1, \ldots, k$, is the line $\eta$ with $\eta_{x y}=\ell_{i}$ and $\eta_{x z}=\lambda_{j}$. By construction $\mu_{i j}$ is a transversal of $S:=R \cup R^{\prime}$ that visits the rectangles of $S$ in the order

$$
\left(r_{k}, \ldots, r_{i+1}, \rho_{j}, \ldots, \rho_{1}, O, \rho_{j+1}, \ldots, \rho_{k}, r_{1}, \ldots, r_{i}\right) .
$$

Thus $\left(r_{i+1}, \rho_{j}\right)$ are geometric-permutation neighbor pairs, for all $i=1, \ldots, k-1$, $j=1, \ldots, k$, and $\rho_{d}(n) \geq \rho_{3}(S) \geq k(k-1)=\Omega\left(n^{2}\right)$, for $d \geq 3$, as claimed.

We now outline the promised transformation of $S$ into a collection of pairwise-disjoint balls with at least as many geometric-permutation neighbor pairs. Observe that, by construction, every ray emanating from $O$ meets the objects of $S$, if at all, consistently with the single linear ordering $\rho_{1}, \rho_{2}, \ldots, \rho_{k}, r_{1}, r_{2}, \ldots, r_{k}$. This means, in particular, that the following procedure will succeed in replacing $S$ by a family $S^{\prime}$ of pairwisedisjoint balls. Start with $\rho_{1}$ and enclose it in a (finite-radius closed) ball that misses the origin. This is possible, since $\rho_{1}$ is a convex set at a positive distance from $O$. Enclose the 
resulting ball $\beta_{1}$ by the smallest ball $\gamma_{1}$ centered at the origin. Now scale $\rho_{2}$ by a positive homothety with center at $O$ until it lies strictly outside of $\gamma_{1}$ to obtain $\rho_{2}^{\prime}$ and replace $\rho_{2}$ by a finite-radius ball $\beta_{2}$ containing $\rho_{2}^{\prime}$ and missing $\gamma_{1}$. Again, enclose $\beta_{2}$ (and $\gamma_{1}$ ) by the smallest ball $\gamma_{2}$ centered at the origin. The process proceeds iteratively. At the $i$ th step we have constructed a ball $\gamma_{i-1}$ centered at $O$ that encloses the already constructed pairwise-disjoint balls $\beta_{1}, \ldots, \beta_{i-1}$. Since the $i$ th rectangle, call it $r$, in the above linear ordering lies at a positive distance from $O$, it can be scaled by a positive homothety with center at $O$ to lie strictly outside of $\gamma_{i-1}$ and then be replaced by a ball $\beta_{i}$ that encloses this scaled version of $r$ and avoids $\gamma_{i-1}$ and thus all the previously constructed balls $\beta_{1}, \ldots, \beta_{i-1}$.

When this process terminates, we obtain a collection $S^{\prime}$ of pairwise-disjoint balls each of which encloses (a positive homothetic copy of) an original rectangle. Hence, a transversal $\ell$ of $S$ is necessarily a transversal of $S^{\prime}$. It remains to argue that we have not changed the permutation corresponding to $\ell$, but this is indeed true, since the newly constructed balls $\beta_{i}$ have the property that they lie in nested concentric spherical shells centered at $O$, in the linear order given above. This completes the proof of the theorem.

Note that, in the above construction, a given pair of objects $a \in R$ and $b \in R^{\prime}$ appear consecutive in only a single permutation. Thus any two objects $x, y$ that appear consecutive in more than one permutation of this construction must belong both to $R$ or both to $R^{\prime}$. The number of such pairs in the above construction is easily seen to be $O(n)$.

At this point, and in view of the above remark, we propose some alternative conjectures to the refuted conjecture of Sharir and Smorodinsky. We replace the notion of "neighbor" by a stronger condition below. Now, for $a, b \in S$, and $k \geq 1$, we call $a$ and $b$ (geometric-permutation) $k$-buddies if $a$ and $b$ are consecutive in at least $k$ distinct geometric permutations. Let $q_{k}(S)$ be the number of $k$-buddy pairs in $S$ and put $q_{k, d}(n)=\max q_{k}(S)$, with the maximum taken over all families $S$ of $n$ pairwise-disjoint objects in $\mathbb{R}^{d}$. Bounding $q_{k, d}(n)$ is useful for bounding the maximum possible number of geometric permutations, as evidenced by the following theorem.

Theorem 3. For any fixed $k \geq 1, g_{d}(n) \leq(k-1)\left(\begin{array}{l}n \\ 2\end{array}\right)+K\left(q_{k, d}(n), d-1\right)=O\left(k n^{2}+\right.$ $\left.q_{k, d}^{d-1}(n)\right)$.

Proof. Consider the set of all geometric permutations $\Pi$ of a suitable family $S$ of $n$ objects in $\mathbb{R}^{d}$ that achieves $q=q_{k, d}(n)$. We "trim" $\Pi$ as follows: while there exists a pair $\{a, b\}$ of objects that occur consecutively in at least one but fewer than $k$ permutations in $\Pi$, discard all the permutations that contain $\{a, b\}$ consecutively. Since any pair of objects can trigger at most $k-1$ such deletions, we have discarded fewer than $(k-1)\left(\begin{array}{l}n \\ 2\end{array}\right)$ permutations.

Let $\Pi^{\prime}$ be the set of remaining permutations; $|\Pi| \leq(k-1)\left(\begin{array}{l}n \\ 2\end{array}\right)+\left|\Pi^{\prime}\right|$. A pair $\{a, b\}$ of consecutive elements in a permutation of $\Pi^{\prime}$ has to appear in at least $k$ distinct permutations in $\Pi$. Thus $a$ and $b$ are $k$-buddies and the number of such pairs is at most $q$. We argue as in [11] and in the proof of Theorem 1 that $\left|\Pi^{\prime}\right| \leq K(q, d-1)$. Indeed, 
pick, for every pair $\{a, b\}$ of $k$-buddies a hyperplane $h=h(a, b)$ separating $a$ from $b$ and let $s=s(a, b) \subset \mathbb{S}^{d-1}$ be the great sphere of directions parallel to $h$. Consider the arrangement $\mathcal{A}$ of the at most $q$ such great spheres. Since no transversal to $S$ is parallel to one of these great spheres, transversal orientations are contained in one of the $K(q, d-1)$ pairs of antipodal cells in $\mathcal{A}$. We argue that orientations from one such antipodal pair can give rise to at most one geometric permutation in $\Pi^{\prime}$. Indeed, fix such a pair $C, C^{\prime}$ of antipodal cells and consider an oriented line transversal of $S$ whose direction lies in $C$ and whose corresponding geometric permutation is in $\Pi^{\prime}$. Any two consecutive objects in the corresponding geometric permutation must be $k$-buddies whose order is determined by our choice of $C$. Hence, there can be at most one permutation of $\Pi^{\prime}$ consistent with the ordering of all $k$-buddy pairs corresponding to $C$. This completes the proof.

Note that Theorem 2 implies $q_{1, d}(n)=\Omega\left(n^{2}\right)$ for $d \geq 3$. Next, we offer the following alternative conjectures to that of Sharir and Smorodinsky:

Strong $\boldsymbol{k}$-Buddies Conjecture. For some fixed $\boldsymbol{k} \geq 2$ and any $d, q_{k, d}(n)=O(n)$.

Weak $\boldsymbol{k}$-Buddies Conjecture. For some fixed $\boldsymbol{k} \geq 2$ and any $d, q_{k, d}(n)=o\left(n^{2}\right)$.

A proof of the former conjecture, for example, together with Theorem 3 would imply that the maximum number of geometric permutations to a family of $n$ disjoint objects in $\mathbb{R}^{d}$ is $\Theta\left(n^{d-1}\right)$.

\section{References}

1. O. Cheong, X. Goaoc, and H.-S. Na. Geometric permutations of disjoint unit spheres. Comput. Geom. Theory Appl., 30:253-270, 2005.

2. H. Edelsbrunner. Algorithms in Combinatorial Geometry. Springer-Verlag, Heidelberg, 1987.

3. H. Edelsbrunner and M. Sharir. The maximum number of ways to stab $n$ convex non-intersecting sets in the plane is $2 n-2$. Discrete Comput. Geom., 5:35-42, 1990.

4. M. Katchalski, T. Lewis, and A. Liu. Geometric permutations and common transversals. Discrete Comput. Geom., 1:371-377, 1986.

5. M. Katchalski, T. Lewis, and A. Liu. The different ways of stabbing disjoint convex sets. Discrete Comput. Geom., 7:197-206, 1992.

6. M. Katchalski, S. Suri, and Y. Zhou. A constant bound for geometric permutations of disjoint unit balls. Discrete Comput. Geom., 29:161-173, 2003.

7. M.J. Katz and K.R. Varadarajan. A tight bound on the number of geometric permutations of convex fat objects in $\mathbb{R}^{d}$. Discrete Comput. Geom., 26:543-548, 2001.

8. M. Sharir and S. Smorodinsky. On neighbors in geometric permutations. Discrete Math., 268:327-335, 2003.

9. S. Smorodinsky. Geometric permutations and common transversals. Master's thesis, School of Mathematical Sciences, Tel-Aviv University, Tel-Aviv, Israel, July 1998.

10. S. Smorodinsky, J.S.B. Mitchell, and M. Sharir. Sharp bounds on geometric permutations of pairwise disjoint balls in $\mathbb{R}^{d}$. Discrete Comput. Geom., 23:247-259, 2000.

11. R. Wenger. Upper bounds on geometric permutations for convex sets. Discrete Comput. Geom., 5:27-33, 1990. 
12. R. Wenger. Progress in geometric transversal theory. In Contemporary Mathematics, B. Chazelle and J.E. Goodman, eds., pp. 375-393. American Mathematical Society, Providence, RI, 1999.

13. R.O. Winder. Partitions of $N$-space by hyperplanes. SIAM Journal on Applied Mathematics, 14:811-818, 1966.

14. Y. Zhou and S. Suri. Shape sensitive geometric permutations. Proceedings of the 12th ACM-SIAM Symposium on Discrete Algorithms, pp. 234-243, 2001.

15. Y. Zhou and S. Suri. Geometric permutations of balls with bounded size disparity. Computational Geometry Theory and Applications, 26:3-20, 2003.

Received December 2, 2003, and in revised form June 6, 2004. Online publication May 27, 2005. 